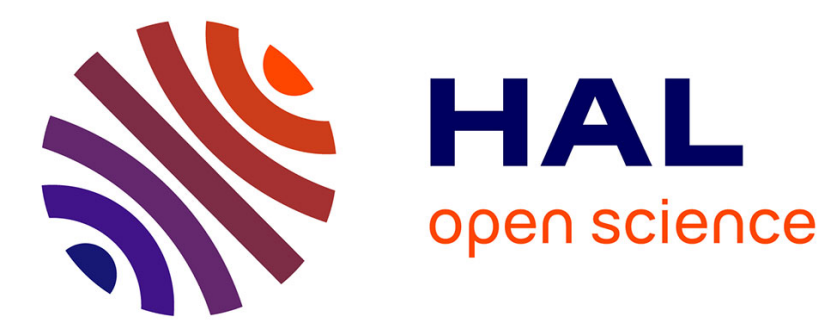

\title{
Class specific elastic, viscous, optical and dielectric properties of some nematic liquid crystals and correlations with their performance in twisted nematic displays \\ M. Schadt, F. Müller
}

\section{To cite this version:}

M. Schadt, F. Müller. Class specific elastic, viscous, optical and dielectric properties of some nematic liquid crystals and correlations with their performance in twisted nematic displays. Revue de Physique Appliquée, 1979, 14 (1), pp.265-274. 10.1051/rphysap:01979001401026500 . jpa-00244586

\author{
HAL Id: jpa-00244586 \\ https://hal.science/jpa-00244586
}

Submitted on 1 Jan 1979

HAL is a multi-disciplinary open access archive for the deposit and dissemination of scientific research documents, whether they are published or not. The documents may come from teaching and research institutions in France or abroad, or from public or private research centers.
L'archive ouverte pluridisciplinaire HAL, est destinée au dépôt et à la diffusion de documents scientifiques de niveau recherche, publiés ou non, émanant des établissements d'enseignement et de recherche français ou étrangers, des laboratoires publics ou privés. 


\title{
Class specific elastic, viscous, optical and dielectric properties of some nematic liquid crystals and correlations with their performance in twisted nematic displays
}

\author{
M. Schadt and F. Müller, \\ Central Research Units, F. Hoffmann-La Roche \& CO. LTD. \\ CH-4002 Basel, Switzerland \\ (Reçu le 10 août 1978, révisé le 9 octobre 1978, accepté le 11 octobre 1978)
}

\begin{abstract}
Résumé. - Nous présentons des résultats expérimentaux des propriétés élastiques, visqueuses, optiques et diélectriques de six mélanges binaires de nématiques à anisotropie diélectrique positive dont les composants appartiennent à 6 classes différentes de cristaux liquides. Le comportement en fonction de la température des constantes du matériel est en bon accord avec la réponse électro-optique d'affichages à structure nématique tordue. Une faible corrélation entre taux de multiplexage de tels affichages et propriétés élastiques a été trouvée. La dépendance de la température des angles d'inclinaison dans des échantillons de cristaux liquides de différentes classes à alignement homogène est mesurée au moyen d'une nouvelle méthode.
\end{abstract}

\begin{abstract}
Measurements are reported of the class specific elastic, viscous, optical and dielectric properties of six positive dielectric binary mixtures consisting of components belonging to six markedly different liquid crystal classes. The temperature dependent material properties are correlated with the temperature dependent response behaviour of twisted nematic displays. The elastic properties were found to be loosely related with the multiplexing performance of TN-LCDs. Good agreement was found between the temperature dependence of material constants and electro-optical response. The temperature dependence of bias tilt angles in homogeneously aligned samples belonging to different LC-classes were measured using a novel method.
\end{abstract}

1. Introduction. - A prerequisite for engineering, characterisation and optimal application of liquid crystal components and mixtures in displays is the knowledge of a number of material parameters and the correlations between them and their electrooptical performance in displays. Some material properties, especially the elastic constants, are difficult to measure and only few data are available for positive dielectric materials [1, 2]. Most measurements of the elastic constants were performed using basically negative dielectric substances such as MBBA or azoxybenzenes, i.e. $[3,4,5]$.

To our knowledge only one attempt has been made so far to measure complete sets of relevant material properties and to relate them with the electro-optical performance of the substances in twisted nematic displays [6]. No attempt has been made to correlate their temperature dependence with the temperature dependent display performance or the chemical class specific properties of the substances in displays.

We shall report on measurements of the temperature dependence of the viscous, dielectric, optical and elastic properties made on six positive dielectric binary nematic mixtures. To identify the class specific properties related with display performance the substances used were chosen among six markedly different liquid crystal classes including a hydrogenated class. The material properties measured will be correlated with the temperature dependence of the electro-optical response of twisted nematic displays and with the class specific multiplexing properties. The influence of surface alignment on bias tilt angle using different liquid crystal materials is investigated by means of a novel measuring method.

2. Experimental. - 2.1 MeAsuring TEChNIQUES. - Measuring the various physical constants which determine the electro-optical performance of twisted nematic displays (TN-LCDs) [7] requires a number of quite different techniques. A prerequisite for some are liquid crystal (LC) samples having well defined boundaries. The experimental methods, the influence of temperature on the surface alignment of different LC-classes and the LC-materials used will be described.

The static dielectric constants $\varepsilon_{\|}, \varepsilon_{\perp}$ in the nematic phase and $\varepsilon_{\text {is }}$ in the isotropic phase were determined in a parallel-plate capacitor with large electrode spac- 
ing ( $1 \mathrm{~mm})$ by means of a magneto-capacitive method described earlier [8]. A rotating cone Brookfield microviscometer [2] (model LVT) was used to measure the bulk viscosity $\eta$.

The ordinary index of refraction $n_{0}$ was measured in unoriented LC-layers with an Abbé refractometer. The extraordinary index of refraction $n_{\mathrm{e}}$ was determined using the recently described $[6,9]$ electricfield induced birefringence method. The interference fringes occurring in the transmission of a homogeneously (parallel) aligned positive dielectric LClayer undergoing a Fréedericksz transition give rise to a number $N$ of transmission maxima. $N$ depends on the optical anisotropy $\Delta n=\left(n_{\mathrm{e}}-n_{0}\right)$, the thickness $d$ of the sample and the wavelength $\lambda$ of transmitted light,

$\delta=\Delta n \cdot d=N \cdot \lambda$.

The optical path difference $\delta$ in equation (1) reaches its maximum at high driving voltages where the transition from homogeneous to homeotropic (perpendicular) alignment is completed. $n_{\mathrm{e}}$ was calculated using equation (1) and the measurements of $n_{0}$ and $N=N_{\max }=$ maximum number of transmission maxima. A He-Ne-laser $(\lambda=632.8 \mathrm{~nm})$ was used as a light source, the display spacing was $d=190 \mu \mathrm{m}$. The thickness of the Mylar spacers used was determined with a micrometer screw and/or via capacitance measurements of the empty cell.

The following assumptions have to be fulfilled for this method to be applicable to determine $n_{\mathrm{e}}$ : (i) the bias tilt angle $\theta$ between the long molecular axes and the electrode surface of the measuring cell is $\theta \cong 0^{\circ}$ in the field-free state (homogeneous alignment) and (ii) the maximum voltage applied to the cell is sufficiently high to induce complete homeotropic alignment. We shall show below that with bias tilt angles $\theta<0.5^{\circ}$ (ii) was fulfilled for all temperatures and materials used in our experiments. The degree of uniaxiality of the field-induced homeotropic alignment was determined by means of conoscopic observation of the samples in white light using a Leitz Orthoplan Pol microscope and fields up to $40 \mathrm{kV} / \mathrm{cm}$. The conoscopic photograph depicted in figure 1 indicates the degree of homeotropic alignment that was achieved. The numerical aperture used for the conoscopic observations was 0.6 . In all materials studied the conoscopically determined off-homeotropic alignment was smaller than $0.2^{\circ}$. Thus, besides (i) also assumption (ii) is fulfilled. The measuring accuracy of $n_{\mathrm{e}}$ is estimated to be $<0.5 \%$ depending mainly on the accuracy with which the electrode spacing $d$ can be determined.

The elastic constants $k_{11}$ (splay) and $k_{33}$ (bend) were determined from differential capacitance measurements on electric-field induced distortions of homogeneously aligned LC-samples using a novel combination of phase-sensitive detection and impedance bridge circuitry [6]. The twist elastic constant $k_{22}$ was determined from the electric field induced optical and capacitive threshold of $90^{\circ}$ twisted nematic displays [6]. As in the birefringence experiments low bias tilt $\left(\theta<0.5^{\circ}\right)$ displays were used. The preparation of the samples was described earlier [2]. The accuracy in the determination of $k_{11}, k_{22}$ and $k_{33}$ is $\pm 3 \%$, $\pm 2 \%$ and $\pm 10 \%$ respectively.

Besides using electric-field induced distortions for determining $k_{11}$ we also measured the magnetic threshold field $H_{0}$ of the Fréedericksz transition in one representative of the cyano biphenyls for which elastic constants were published recently [1]. $k_{11}$ was calculated from $H_{0}$ and measurements of the magnetic susceptibility $\Delta \chi=\left(\chi_{\|}-\chi_{\perp}\right)$. The Faraday method was used to determine $\Delta \chi$.

The apparatus for measuring the static and dynamic transmission of TN-LCDs (90 twist angle) was described recently [6]. The static measurements were performed in low bias tilt angle displays $\left(\theta<0.5^{\circ}\right)$, whereas displays with high bias tilt angles $\left(\theta \sim 22^{\circ}\right)$ were used for the dynamic measurements. The exact spacing $d(\sim 11 \mu \mathrm{m})$ was determined from measurements of the capacity of the empty displays. Small variations of $d$ were accounted for by normalizing the results of the dynamic experiments to $d=11 \mu \mathrm{m}$ by making use of the proportionality $t_{\text {on }}, t_{\text {off }} \propto d^{2}$.

In all experiments requiring wall oriented LCsamples alignment was achieved by angular evaporation of $\mathrm{SiO}_{x}$ [10] onto conducting, $3 \mathrm{~mm}$ thick glass plates. A $30^{\circ}$ angle of evaporation was used to get low bias tilt surfaces $\left(\theta<0.5^{\circ}\right)$; whereas high bias tilt surfaces were obtained by using an angle of evaporation of $5^{\circ}\left(19^{\circ}<\theta<25^{\circ}\right)$.

The bias tilt angle $\theta$ was measured by means of the magneto-capacitive null method described by Toriyama and Ishibashi [11] and by Scheffer and Nehring [12] as well as by a novel electro-capacitive method based on determining the dielectric constant $\varepsilon_{\mathrm{d}}$ of a tilted LC-layer in a display. $\varepsilon_{\mathrm{d}}$ depends on the dielectric constants $\varepsilon_{\perp}$ and $\varepsilon_{\|}$of the LC-material as well as on the bias tilt angle $\theta$. Assuming the principal dielectric axes $\varepsilon_{\|}$to be parallel to the nematic director - an assumption which the optical experiments showed to be correct for the materials investigated (Fig. 1) $\varepsilon_{\mathrm{d}}$ is given by

$\varepsilon_{\mathrm{d}}=\varepsilon_{\|} \sin ^{2} \theta+\varepsilon_{\perp} \cos ^{2} \theta$.

Equation (2) reduces in the isotropic phase to

$\varepsilon_{\mathrm{d}}(\mathrm{is})=\varepsilon_{\text {is }}$.

$\varepsilon_{\mathrm{d}}$ was measured in parallel aligned displays with $1 \mathrm{~cm}^{2}$ electrodes and $19 \mu \mathrm{m}$ spacers. To avoid erroneous measurements due to stray capacities, inaccurate display spacings and electrode areas, $\varepsilon_{\mathrm{d}}$ (is) was determined for each display and compared with $\varepsilon_{i s}$. The accuracy of the method depends mainly on the accuracy with which the dielectric constants 


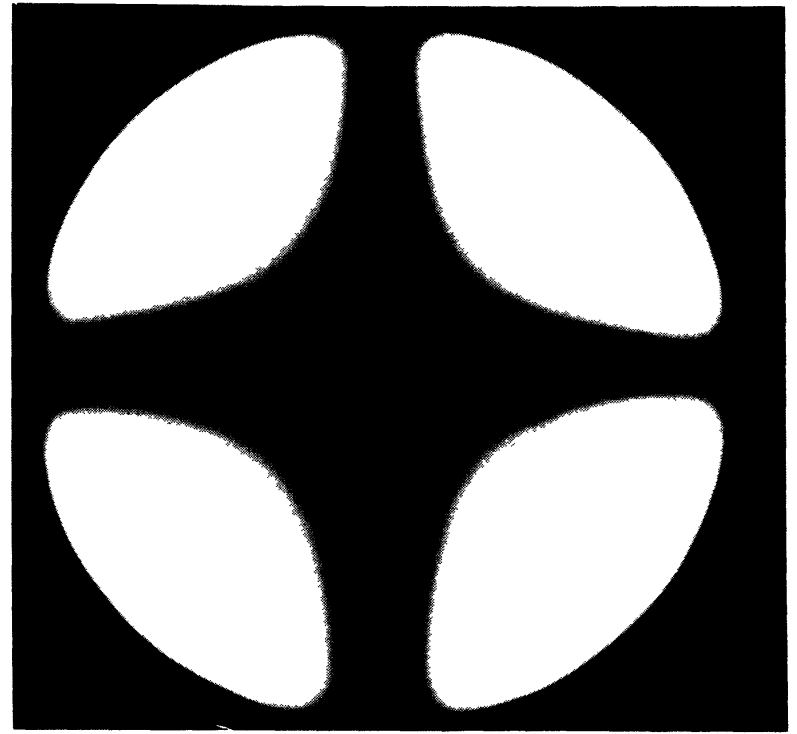

Fig. 1. - Conoscopic picture made of electric-field induced homeotropic alignment using RO-TN-200 and a field of $40 \mathrm{kV} / \mathrm{cm}$.

can be determined. With an error of $< \pm 0.5 \%$ in the determination of $\varepsilon$ we assume $\theta=20^{\circ} \pm 0.1^{\circ}$. From equation (2) and $\varepsilon_{\mathrm{d}} \rightarrow \varepsilon_{\perp}$ follows that the accuracy of the method decreases with decreasing $\theta$. Thus, the capacitive null method [11] which is more accurate for determining low bias tilt angles was used for $\theta<5^{\circ}$.

Figure 2 shows results obtained from electrocapacitive measurements of the temperature dependence of the bias tilt angle performed in $5^{\circ}$ angular evaporated displays. Three mixtures belonging to different LC-classes namely cyano biphenyls (ROTN-570), cyano Schiff' bases (RO-TN-200) and cyano

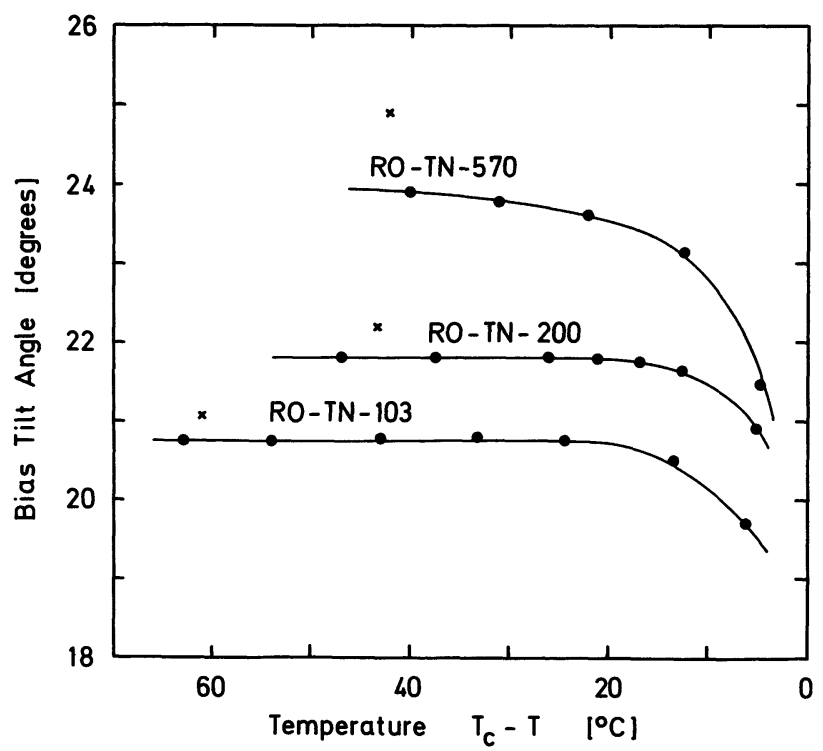

Fig. 2. - Temperature dependence of bias tilt angle $\theta$ measured by the electro-capacitive method for three different LC-materials using $5^{\circ}$ angular evaporated electrodes. The measurements designated $x$ were made using the magneto-capacitive null method. esters (RO-TN-103) from F. Hoffmann-La Roche were investigated. The dielectric constants and compositions of RO-TN-200 and RO-TN-103 were published before $[6,13]$. The bias tilt angle $\theta$ decreases with increasing temperature when using high bias tilt electrode surfaces (Fig. 2). The strongest decrease of $\theta$ occurs in the biphenyl mixture and at temperature $T \rightarrow T_{\mathrm{c}}$ (Fig. 2). This finding is in agreement with those of Toda et al. [14] who investigated the biphenyl mixture E-7 from British Drug Houses which is virtually identical to RO-TN-570. The smaller temperatures dependence $\theta(T)$ measured for Schiff' bases and esters (Fig. 2) may reflect their stronger tendency for homogeneous alignment compared with pure biphenyls. The measurements in figure 2 show that the temperature dependence of bias tilt can be neglected for $\left(T_{c}-T\right)>5{ }^{\circ} \mathrm{C}$ in experiments using high bias tilt displays.

The measurements designated $x$ in figure 2 were made for comparison using the magneto-capacitive null method [11]. The electro-capacitive method seems to give slightly lower bias tilt angles than the magnetocapacitive method (Fig. 2). The reason for the discrepancy is not yet clear.

We did not find any temperature dependence of $\theta$ when using low bias tilt angle displays (evaporation angle $30^{\circ}$ ). This result agrees with others $[14,15]$. It shows that variations of bias tilt with temperature have not to be taken into account in experiments where angular evaporated low bias tilt $\left(\theta<0.5^{\circ}\right)$ electrode surfaces are used. However, this finding may not be applicable to negative dielectric LC-materials as measurements of bias tilt angles in homogeneously aligned samples of alkylazoxybenzenes showed. Using these materials in $30^{\circ}$ angular evaporated cells we did not obtain zero bias tilt as with positive dielectric substances, but $\theta=22.5^{\circ}$ at room temperature. Thus, bias tilt angles should always be determined when performing experiments requiring defined boundaries.

2.2 Materials. - Six positive dielectric binary liquid crystal mixtures were investigated consisting of components that belong to 2-paracyanophenylalkylpyrimidines [16] $P_{3} X$, cyano Schiff bases [16] SX, cyano esters [16] EX, cyano alkyl biphenyls [17] BX, cyano alkoxy biphenyls [17] BOX and p-cyanophenyl-4-alkyl-cyclohexanecarboxylates [18] PCHEX respectively. $\mathrm{X}$ designates the number of alkyl carbon atoms. The mixtures designated $a=\left(\mathrm{P}_{3} 5, \mathrm{P}_{3} 7\right)$, $b=$ (S5, S7), $c=$ (E5, E7), $d=$ (B5, B7), $e=(\mathrm{BO} 5$, BO7) and $f=($ PCHE5, PCHE7) contained each a pentyl and a heptyl component in molar proportions $40 \%: 60 \%$. Binary mixtures were chosen instead from single components because of their larger mesomorphic range and their stronger supercoolability. All the components were synthesized by F. Hoffmann-La Roche. The phenylcyclohexane esters in mixture $f$ that were first described by Demus et al. [18] 
Table I. - Measurements of the nematic-isotropic transition temperature $T_{\mathrm{c}}$ of mixtures a $-f . \Delta \varepsilon$ and $\varepsilon_{\perp}$ were measured at $\left(T_{\mathrm{c}}-T\right)=20^{\circ} \mathrm{C} . n_{0}, n=\left(n_{\mathrm{e}}-n_{0}\right), k_{11}, k_{22}, k_{33}$ and $k_{33} / k_{11}$ were measured at constant reduced temperature $T / T_{\mathrm{c}}=0.95$. The bulk viscosity $\eta$ was measured at constant temperature $T=30{ }^{\circ} \mathrm{C}$. $A=$ activation energy of $\eta(T)$.

\begin{tabular}{|c|c|c|c|c|c|c|c|c|c|c|c|}
\hline Mixture & $\begin{array}{c}T_{\mathrm{c}} \\
\left({ }^{\circ} \mathrm{C}\right)\end{array}$ & $\Delta \varepsilon$ & $\varepsilon_{\perp}$ & $n_{0}$ & $\Delta n$ & $\begin{array}{c}\eta \\
\text { (cP) }\end{array}$ & $\begin{array}{c}A \\
(\mathrm{eV})\end{array}$ & $\begin{array}{c}k_{11} \\
\left(\times 10^{-12} \mathrm{~N}\right)\end{array}$ & $\begin{array}{c}k_{22} \\
\left(\times 10^{-12} \mathrm{~N}\right)\end{array}$ & $\begin{array}{c}k_{33} \\
\left(\times 10^{-12} \mathrm{~N}\right)\end{array}$ & $k_{33} / k_{11}$ \\
\hline - & - & - & - & - & - & - & - & - & - & - & - \\
\hline$a$ & 51.0 & 22.6 & 7.7 & 1.5141 & 0.18 & 33.0 & 0.376 & 8.0 & 6.1 & 8.7 & 1.07 \\
\hline$b$ & 71.7 & 13.9 & 6.9 & 1.5255 & 0.22 & 48.5 & 0.463 & 7.5 & 6.6 & 14.6 & 1.82 \\
\hline$c$ & 56.2 & 21.7 & 7.9 & 1.5031 & 0.16 & 54.0 & 0.489 & 7.1 & 5.9 & 13.7 & 1.93 \\
\hline$d$ & 39.5 & 13.0 & 5.5 & 1.5240 & 0.19 & 22.5 & 0.407 & 7.9 & 7.8 & 15.4 & 1.94 \\
\hline$e$ & 71.7 & 11.0 & 6.4 & 1.5185 & 0.19 & 58.0 & 0.477 & 6.0 & 6.2 & 11.5 & 1.89 \\
\hline$f$ & 79.7 & 7.30 & 5.8 & 1.4700 & 0.09 & 34.0 & 0.387 & 6.8 & 6.4 & 15.2 & 2.22 \\
\hline
\end{tabular}

are the only ones containing a hydrogenated ring. The nematic-isotropic transition temperatures of the mixtures are depicted in Table $I$.

3. Material properties. -3.1 BULK VISCOSITY. Figure 3 shows two plots of the temperature dependence of the bulk viscosity $\eta$. The right hand figure demonstrates that $\eta(T)$ can in all cases be approximated by

$\eta \propto \exp (A / k T)$

where $A$ is an activation energy which is constant over most of the mesomorphic ranges (Fig. 3). It is interesting to note that the activation energies of all mixtures exceed $0.4 \mathrm{eV}$ except for the pyrimidine mixture $a$ and the PCHE-mixture $f$ for which $A<0.4 \mathrm{eV}$ was found (Table I). The lowest bulk viscosity was measured with the alkyl biphenyl mixture $d$, followed by pyrimidines $(a)$, cyclohexane esters $(f)$, Schiff' bases $(b)$, esters (c) and alkoxy biphenyls (e) respectively (Fig. 3, Table I). The results of mixture e show that alkoxy groups increase $\eta$ considerably, whereas hydration leads to a considerable reduction; c.f. hydrated and nonhydrated ester mixtures $f$ and $c$ respectively. This finding is in agreement with the scaling down of the bulk viscosity of phenylcyclohexanes [19] compared with biphenyls. It appears that the intro-

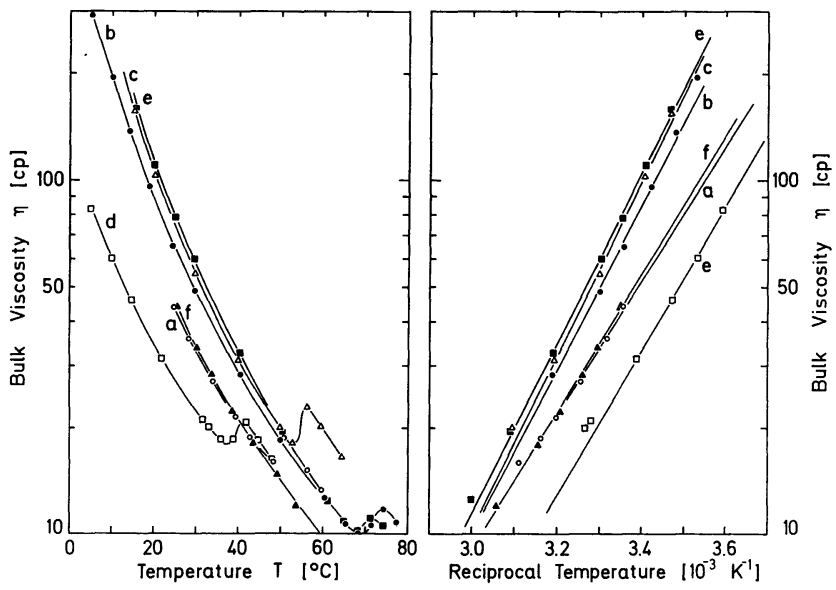

Fig. 3. - Two representations of the temperature dependence of the bulk viscosity $\eta$ measured for binary mixtures $a-f$. duction of a hydrogenated ring causes the viscosity to decrease by approximately a factor of 1.6 compared with $\eta$ of the analogous aromatic LC-class.

3.2 StatiC DiEleCtriC CONSTANTS. - The measurements depicted in figure 4 show the temperature dependence of the static dielectric anisotropy $\Delta \varepsilon=\left(\varepsilon_{\|}-\varepsilon_{\perp}\right)$ and $\varepsilon_{\perp}$ of the binary mixtures $a-f$. For comparison table I shows the dielectric constants measured at constant temperature $\left(T_{\mathrm{c}}-T\right)=20^{\circ} \mathrm{C}$. All substances exhibit a positive dielectric anisotropy,

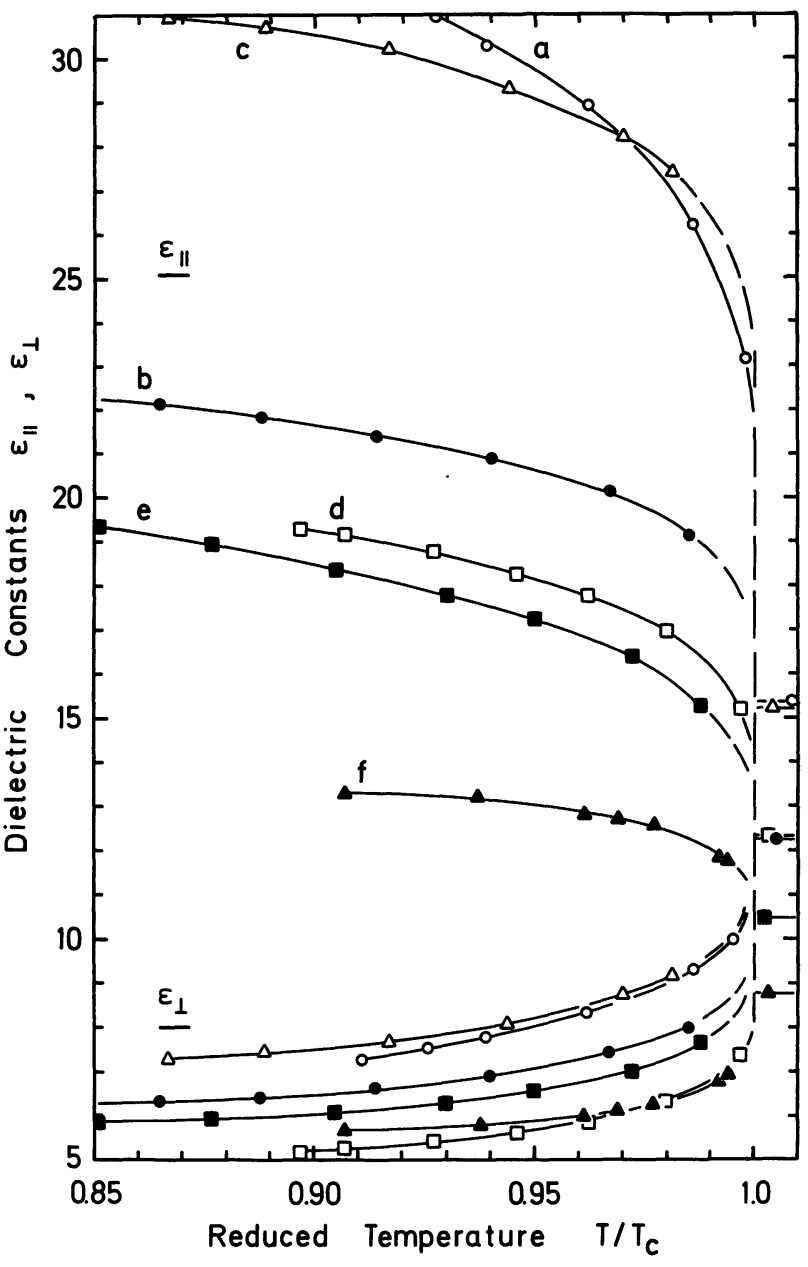

Fig. 4. - Temperature dependence of the static dielectric constants $\varepsilon_{\|}$and $\varepsilon_{\perp}$. 
which is most pronounced for the pyrimidine mixture $a$ and ester mixture $c$ (Fig. 4, table I). Both materials have - in addition to the common cyano end group strong permanent dipole components along their long molecular axes; i.e. the pyrimidine nitrogens and the ester linkage respectively [16]. The Schiff' base as well as the biphenyl mixtures exhibit smaller dielectric anisotropies, whereas $\Delta \varepsilon$ of the hydrated mixture $\mathrm{f}$ is astonishingly small (Fig. 4, Table $\mathrm{I}$ ).

The small dielectric anisotropy of mixture $f$ cannot only be attributed to the reduction of the conjugated $\pi$-electron system due to hydration of one benzene ring in PCHEs. If it is assumed that the directions and magnitudes of the permanent dipole moments in aromatic esters $\mathrm{E}$ - which are mainly responsible for their large $\Delta \varepsilon-$ are comparable to those in PCHEs one should not expect the latter to exhibit the observed low $\Delta \varepsilon$ values. According to Maier and Meier [20] $\Delta \varepsilon$ is given by

$\frac{\Delta \varepsilon}{4 \pi}=N h F\left[\Delta \alpha-F \frac{\mu^{2}}{2 k T}\left(1-\cos ^{2} \beta\right)\right] S$.

$N \cong$ Avogadro's number, $h=$ cavity field factor of the empty cavity, $F=$ reaction field factor, $\Delta \alpha=\left(\alpha_{\|}-\alpha_{\perp}\right)=$ anisotropy of induced polarizability, $\mu=$ permanent dipole moment, $\beta=$ angle between $\mu$ and long molecular axes and

$S=\left\langle 1-\frac{3}{2} \sin ^{2} \theta\right\rangle=$ long range order parameter .

Under the above made assumptions the second term in the brackets of equation (5) should be comparable for Es and PCHEs, whereas $\Delta \alpha$ decreases upon hydrogenation. The decrease of $\Delta \alpha$ in PCHEs is significant as measurements of the optical anisotropies will show. However, because of its minor contribution to $\varepsilon_{\|}$ it cannot account for their low dielectric anisotropy. Yet, it may well be that the cavity field factor $h$ and/or the reaction field factor $F(5)$ differ considerably in hydrogenated components compared with their aromatic analogues.

3.3 ReFRACTIVE INDICES. - Figure 5 shows measurements of the temperature dependence of the ordinary and extraordinary indices of refraction. The largest optical anisotropies were those of mixtures containing components with elongated and highly conjugated $\pi$-electron systems such as Schiff' bases (mixture $b$ in figure 5). Smaller values of

$\Delta n=\left(n_{\mathrm{e}}-n_{0}\right)$

were measured in mixtures consisting of highly conjugated but shorter biphenyls and of the optically similar pyrimidines (mixtures $d, e$ and $a$ respectively in figure 5). Still smaller optical anisotropies were measured in mixture $c$ containing the elongated but relatively weakly conjugated esters. The smallest values of $\Delta n$ were those of mixture $f$ containing PCHE whose hydrogenated rings do hardly contribute to the optical

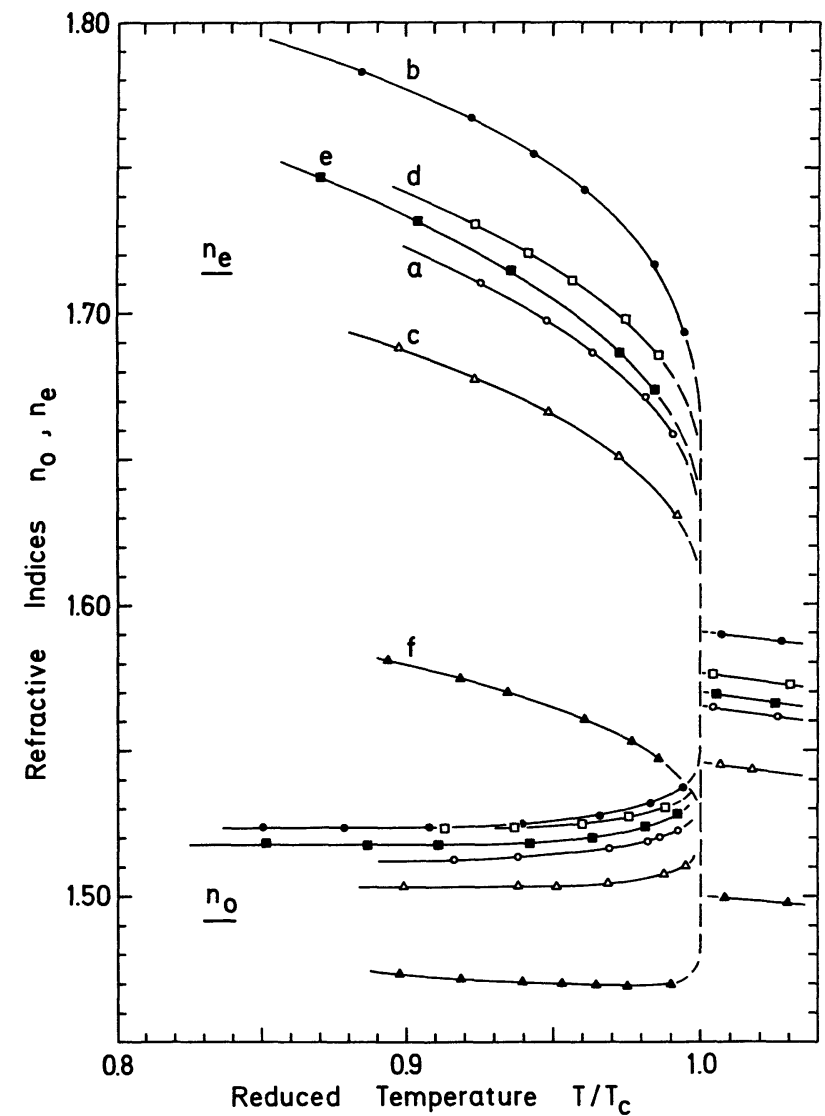

Fig. 5. - Temperature dependence of the ordinary $\left(n_{0}\right)$ and extraordinary $\left(n_{\mathrm{e}}\right)$ indices of refraction.

anisotropy of the molecules. $\Delta n$ of PCHE is mainly determined by the optical anisotropy of the benzene ring and - to a lesser extent - by the ester linkage. Table I shows measurements of $n_{0}$ and $\Delta n$ made at constant reduced temperature

$T / T_{\mathrm{c}}=0.95$.

3. 4 Elastic CONSTANTS. - The twist elastic constant $k_{22}$ was calculated from

$V_{\mathrm{t}}=\pi\left[\frac{1}{\Delta \varepsilon \varepsilon_{0}}\left[k_{11}+\frac{\left(k_{33}-2 k_{22}\right)}{4}\right]\right]^{1 / 2}$

using the measurements of $k_{11}, k_{33}$ (Fig. 6) and $\Delta \varepsilon$ (Fig. 4). The threshold voltage $V_{t}$ in equation (6) was determined by measuring the actual optical threshold of $19 \mu \mathrm{m}$ thick TN-LCDs [6]. $k_{11}$ was determined from the threshold voltage $V_{c}$ of the Fréedericksz transition of homogeneously aligned samples

$V_{\mathrm{c}}=\pi\left(k_{11} / \varepsilon_{0} \Delta \varepsilon\right)^{1 / 2}$,

whereas $k_{33}$ was determined from measurements of electric field-induced capacitance changes $\Delta C$ occurring in homogeneously aligned samples using [6]

$\Delta C \propto \frac{2 \gamma\left(V_{1} / V_{c}-1\right)}{1+\gamma+\kappa}$. 


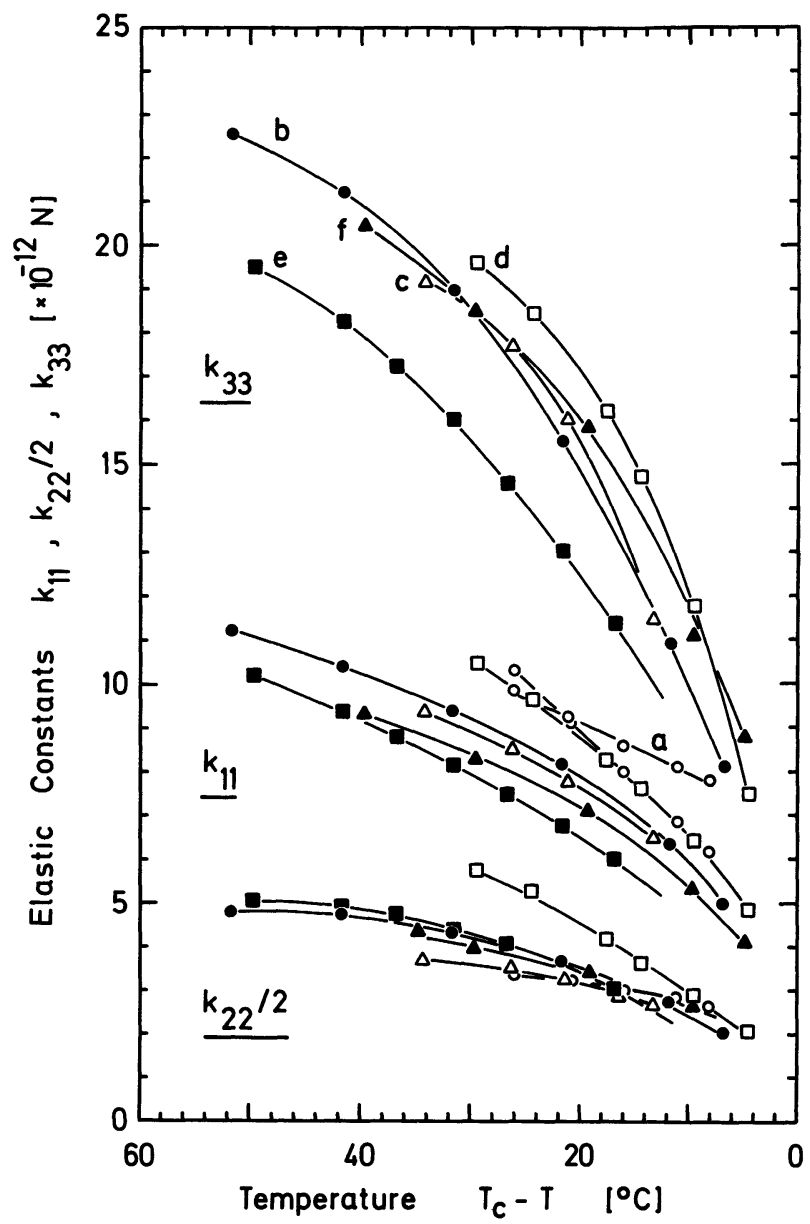

Fig. 6. - Temperature dependence of the splay $\left(k_{11}\right)$, twist $\left(k_{22}\right)$ and bend $\left(k_{33}\right)$ elastic constants of mixtures $a-f$. For reasons of clarity $k_{22} / 2$ is depicted.

$\kappa=\left(k_{33}-k_{11}\right) / k_{11}, \gamma=\Delta \varepsilon / \varepsilon_{\perp}$ and $V_{1}=$ aligning voltage. A detailed description of the method and the evaluation of the results was published recently [6].

The measurements depicted in figure 6 show the temperature dependence of the splay $\left(k_{11}\right)$, twist $\left(k_{22}\right)$ and bend $\left(k_{33}\right)$ elastic constants of mixtures $a-f$. For reasons of clearity $k_{22} / 2$ is depicted in the graphs of figure 6 . The values of $k_{\mathrm{ii}}$ and $k_{33} / k_{11}$ measured at constant reduced temperature $T / T_{\mathrm{c}}=0.95$ are shown in table I. Figure 7 shows the temperature dependence of the ratio $k_{33} / k_{11}$ derived from the measurements in figure 6 . It is interesting to note that

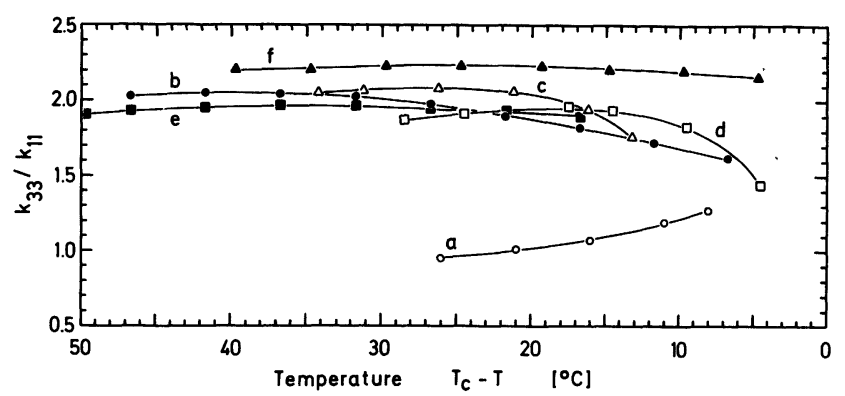

Fig. 7. - Temperature dependence of the elastic ratio $k_{33} / k_{11}$ determined from the measurements depicted in figure 6 . the variations of the elastic constants and of $k_{33} / k_{11}$ among markedly different LC-classes dot not vary much (Figs. 6, 7); they are much smaller than those found by de Jeu and Claassen within homologous series of azoxybenzenes [4] for instance.

The molecular statistical theory of Maier and Saupe [21] predicts the elastic constants to be proportional to $S^{2}, S$ being the long range order parameter. Thus, the ratio $k_{33} / k_{11}$ should be constant at all temperatures. However, figure 7 shows that this is not the case for any of the mixtures investigated. A similar finding was reported recently for a homologous series of azoxybenzenes [3]. Over most of the mesophases of mixture $b-f$ it was found

$$
1.5<k_{33} / k_{11}<2.1
$$

(Fig. 7). The only exception was pyrimidine mixture $a$ which exhibits considerably smaller ratios

$0.9<k_{33} / k_{11}<1.4$

(Fig. 7). It will be shown further on that the ratio $k_{33} / k_{11}$ can indeed be loosely related to the multiplexability of liquid crystals. An assumption which has so far not been verified experimentally.

The values of $k_{11}$ and $k_{33}$ measured for the biphenyl mixtures $d$ and $e$ (Fig. 6) appear to be low compared with those reported by Karat et al. who determined $k_{11}$ and $k_{33}$ of the homologous cyano biphenyl series [1]. These authors determined $k_{11}$ from measurements of the magnetic threshold field $H_{0}$ in homogeneously aligned samples using estimated values for $\Delta \chi$. From

$k_{11}=\Delta \chi H_{0}^{2} d^{2} / \pi^{2}$

they obtained for $4^{\prime}$-heptyl-4-cyanobiphenyl at $21.9^{\circ} \mathrm{C}$ $k_{11}=17.5 \times 10^{-12} \mathrm{~N}$. The estimated value for the anisotropic diamagnetic volume susceptibility used was $\Delta \chi=2.34 \times 10^{-7}[1]$. We measured $\Delta \chi$ at $21.9^{\circ} \mathrm{C}$ obtaining $\Delta \chi=1.109 \times 10^{-7}$. Using this value together with $H_{0}=700.0$ gauss measured at $21.9{ }^{\circ} \mathrm{C}$ we obtain from equation (9)

$k_{11}=8.6 \times 10^{-12} \mathrm{~N}$.

Thus, our measurements show that the estimated value for $\Delta \chi$ used by Karat $e t a l$. is by a factor of 2.11 too high leading to the reported high values for $k_{11}$ and $k_{33}$.

\section{Correlations between electro-optical perfor-} mance of TN-LCDs and material properties. - 4.1 MultiPleXABILITY. - The design of TN-LCDs which are multiplexable over a given temperature range requires optimization of both display technology and LC-material properties. The difficulties connected with the optimization increase considerably with increasing number of scanned lines and/or operating temperature range. To achieve good results LC-mixtures containing suitable components have to be chosen; 
i.e. components inducing steep transmission characteristics exhibiting a low temperature dependence as well as a wide field of view and fast electro-optical response. In the following we shall discuss the classspecific dependence of the maximum number of scanned lines based on experiments performed with the binary mixtures $a-f$. An attempt will be made to correlate multiplexability with their elastic and dielectric constants.

Alt and Pleshko [22] derived the dependence of the maximum number of multiplexable lines of a TNLCD matrix on the slope of the static transmission characteristic. To characterize the slope they defined a device parameter $p=\Delta / V_{10}$, with

$\Delta=\left(V_{\text {on }}-V_{10}\right)$.

$V_{\text {on }}=$ driving voltage for which the display is acceptably transmissive. The maximum number $N_{\max }$ of scanned lines increases with increasing slope according to [22]

$N_{\max }=\left[\frac{(1+p)^{2}+1}{(1+p)^{2}-1}\right]^{2}$.

$p$ in equation (10) depends strongly on viewing and bias tilt angle of the display [22] as well as on operating temperature and nematic-isotropic transition temperature $T_{\mathrm{c}}$ [6]. In the ideal multiplexing case where $N_{\max }$ becomes infinite the static transmission of the display approaches a step function, i.e. $p=0$ in equation (10).

Table II shows measurements of the operating voltages $V_{10}$ and $V_{90}$ of mixtures $a-f$ in $\mathrm{TN}$ LCDs. $V_{10}$ is defined as the voltage at which the transmission of the display is $10 \%$ (using parallel polarizers), whereas $V_{90}$ was measured at $90 \%$ transmission. $V_{10}$ and $V_{90}$ in table II are averaged values obtained from at least six measurements made in different low bias tilt $\left(0<0.5^{\circ}\right)$ TN-LCDs. To exclude the influence of $T_{\mathrm{c}}$ on $N_{\max }$ [6] the measurements were made at constant temperature

$\left(T_{\mathrm{c}}-T\right)=20^{\circ} \mathrm{C}$ using vertically incident He-Ne-laser light. $N_{\max }$, depicted in table II, was calculated from equation (10) using the measurements of $V_{10}$ and $V_{\text {on }}=V_{90}$. Using $V_{\text {on }}=V_{50}$ instead of $V_{\text {on }}=V_{90}$ caused $N_{\max }$ to increase due to a reduction of $p$ in equation (9), however, the relative multiplexing performance of the different mixtures did not change. Also shown in table II are values of the elastic constants $k_{11}, k_{33}$, $k$ and the ratio $k_{33} / k_{11}$ determined from the measurements made at $\left(T_{\mathrm{c}}-T\right)=20^{\circ} \mathrm{C}$ (Fig. 7).

The largest number of multiplexable lines were those obtained using pyrimidine mixture $a$, followed by mixtures $b, d, f, e$ and $c$ respectively (Table II). A comparison of $N_{\max }$ with $\Delta \varepsilon / \varepsilon_{\perp}$ (Table II) calculated from $\Delta \varepsilon$ and $\varepsilon_{\perp}$ depicted in table I shows no correlation between the dielectric ratio and the multiplexability of mixtures $a-f$. This finding is in contrast to a suggestion recently made by Kmetz according to which the multiplexability of LC-materials should improve with decreasing $\Delta \varepsilon / \varepsilon_{\perp}$ [23]. If this were correct mixtures $a$ and $c$ - which both exhibit the highest ratio $\Delta \varepsilon / \varepsilon_{\perp}$ (Table I) - should have poor multiplexing properties, in contrast to the results obtained for $N_{\max }$ (Table II). Our findings are not surprising considering the complex field-dependence of the deformation of the nematic director in TN-LCDs on elastic as well as dielectric properties [24].

A comparison of the splay/bend ratio $R=k_{33} / k_{11}$ with $N_{\max }$ (Table II) indicates the existence of a correlation between multiplexability and ratio $R$. The largest value for $N_{\max }$ was found in mixture a for which the smallest ratio $R$ was determined (Table II). $N_{\max }$ of mixtures $a-e$ decreased in the same order as the ratio $R$ increased (Table II), i.e. $a \rightarrow b \rightarrow d \rightarrow e \rightarrow c$. This result is qualitatively in agreement with the expected increase of the slope of the electro-optical transmission of homogeneously aligned LC-samples with decreasing ratio $k_{33} / k_{11}$. Whereby the increasing slope corresponds to an improved multiplexability according to equation (10). The only exception to this rule was the hydrogenated mixture $f$ which did not exhibit the lowest value $N_{\max }$

Table II. - All measurements on mixtures $a-f$ depicted in table II were made at constant temperature $\left(T_{\mathrm{c}}-T\right)=20^{\circ} \mathrm{C}$.

$V_{10}, V_{90}=$ static driving voltage of TN-LCDs ( $r m s$ values) measured at $10 \%$ and $90 \%$ transmission respectively, $N_{\max }=$ maximum number of multiplexable lines, $k=\left(k_{11}+\left(k_{33}-2 k_{22}\right) / 4\right)$.

\begin{tabular}{|c|c|c|c|c|c|c|c|c|c|}
\hline Mixture & $\begin{array}{c}k_{11} \\
\left(\times 10^{-12} \mathrm{~N}\right)\end{array}$ & $\begin{array}{c}k_{33} \\
\left(\times 10^{-12} \mathrm{~N}\right)\end{array}$ & $\begin{array}{c}k \\
\left(\times 10^{-12} \mathrm{~N}\right)\end{array}$ & $k_{33} / k_{11}$ & $\frac{\Delta \varepsilon}{\varepsilon_{\perp}}$ & $\begin{array}{c}\eta \\
\text { (cp) }\end{array}$ & $\begin{array}{l}V_{10} \\
(\mathrm{~V})\end{array}$ & $\begin{array}{l}V_{90} \\
(\mathrm{~V})\end{array}$ & $N_{\max }$ \\
\hline - & - & - & - & - & - & - & - & - & - \\
\hline$a$ & 8.9 & 9.1 & 8.05 & 1.02 & 2.94 & 32.2 & 1.047 & 1.427 & 11.1 \\
\hline$b$ & 7.9 & 14.8 & 8.12 & 1.87 & 2.01 & 17.1 & 1.382 & 1.890 & 10.9 \\
\hline$c$ & 7.6 & 15.5 & 8.30 & 2.04 & 2.75 & 37.8 & 1.080 & 1.609 & 7.0 \\
\hline$d$ & 8.8 & 17.1 & 8.45 & 1.94 & 2.36 & 35.5 & 1.463 & 2.054 & 9.4 \\
\hline$e$ & 6.5 & 12.4 & 6.22 & 1.91 & 1.72 & 18.1 & 1.217 & 1.784 & 7.4 \\
\hline$f$ & 7.2 & 16.0 & 7.72 & 2.22 & 1.26 & 9.8 & 1.576 & 2.248 & 8.6 \\
\hline
\end{tabular}


despite its highest ratio $R=2.23$ (Table II). This may be due to the low birefringence of mixture $f$ which - as Meyerhofer has shown experimentally [28] may lead to steeper transmission curves; i.e. to higher multiplexing ratios $N_{\max }$.

4.2 Electro-optical Response. - The few attempts made so far to correlate LC-material properties with their electro-optical response in TNLCDs were mainly restricted to negative dielectric MBBA doped with some positive dielectric additive to achieve mixtures with $\Delta \varepsilon>0[25,26]$. To our knowledge there was only one attempt to correlate complete sets of material constants, i.e. $\Delta \varepsilon, \eta$ and $k_{i i}$, with the performance in TN-LCDs using positive dielectric substances [6]. In this paragraph we shall extend our previous correlations between electrooptical response of TN-LCDs at room temperature and material properties [6] over the whole mesomorphic range using the LC-class-specific binary mixtures $a-f$.

For the correlations we use the model of Jakeman and Raynes [27] who derived analytical expressions for the turn-on $\left(t_{\mathrm{on}}\right)$ and turn-off $\left(t_{\text {off }}\right)$ times of TNLCDs. This model was derived for small angles of

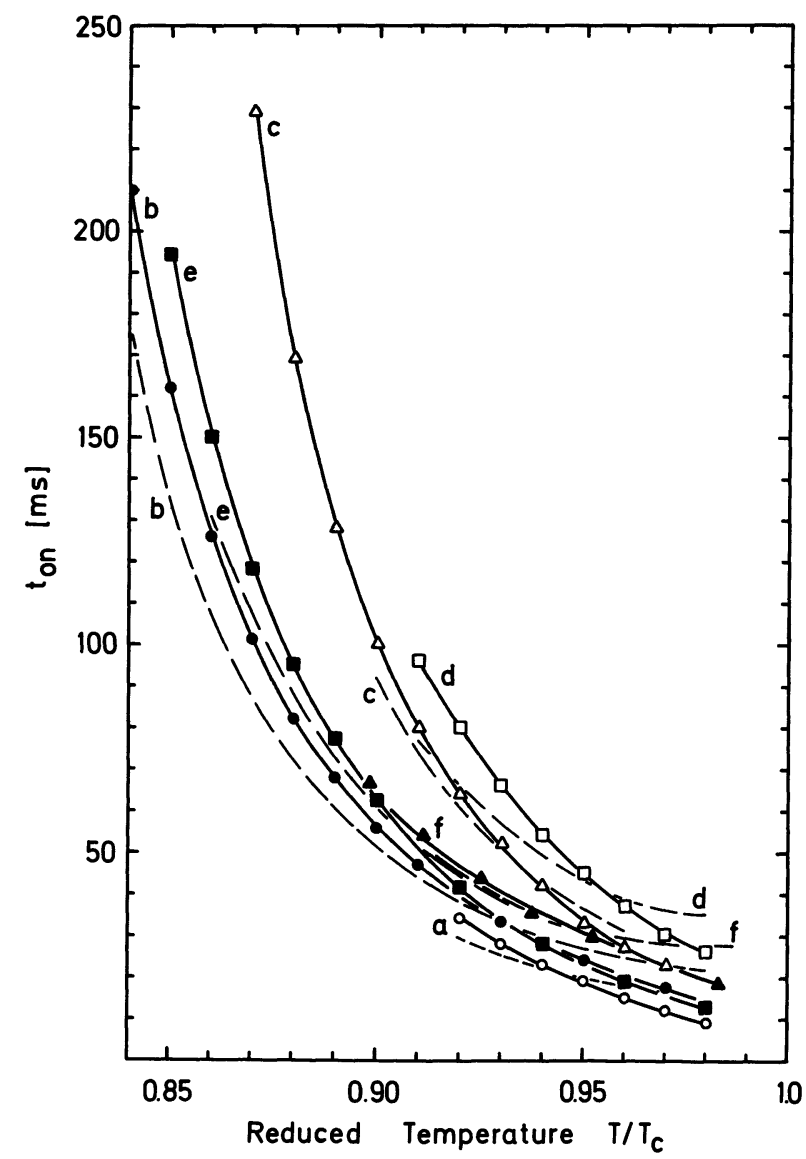

Fig. 8. - Measured (solid lines) and calculated (dotted lines) temperature dependence of the turn-on times of TN-LCDs using mixtures $a-f$. The measurements were made at constant driving voltage ( 3 volts $\mathrm{rms})$ using $11 \mu \mathrm{m}$ displays. The calculated graphs where obtained from the measurements $\eta, \Delta \varepsilon$ and $k_{i i}$ using equation (11) and the scaling factors $f_{\text {on }}$ depicted in table III. deformation of the nematic director only. Driving voltage dependent backflow giving rise to secondary maxima in the decay transmission characteristics of TN-LCDs was not taken into account, nor was the influence of bias tilt on the dynamics [6]. $t_{\text {on }}$ and $t_{\text {off }}$ are given by

$t_{\text {on }}=\eta /\left(\varepsilon_{0} \Delta \varepsilon E^{2}-k \dot{q}^{2}\right)$

and

$t_{\text {off }}=\eta / k q^{2}$.

$t_{\text {on }}$ is defined here as the time required for the transmission to reach $90 \%$ after the driving voltage has been applied, whereas $t_{\text {off }}$ is the time required for the transmission to reach $10 \%$ after the driving voltage has been switched off. $k=\left(k_{11}+\left(k_{33}-2 k_{22}\right) / 4\right)$, $\eta=$ twist viscosity being approximated here by the bulk viscosity, $E=$ electric driving field and $q=\pi / d=$ wave vector of the nematic twist.

The graphs depicted in figures 8 and 9 show $t_{\text {on }}$ and $t_{\text {off }}$ measured in high bias tilt TN-LCDs (solid lines) using mixtures $a-f$. The dotted graphs in figures 8 and 9 show the calculated temperature dependence. The measurements were made at constant driving voltage (3 volts rms) using a gated $90 \mathrm{~Hz}$ sine-wave generator with infinite duty cycle [6].

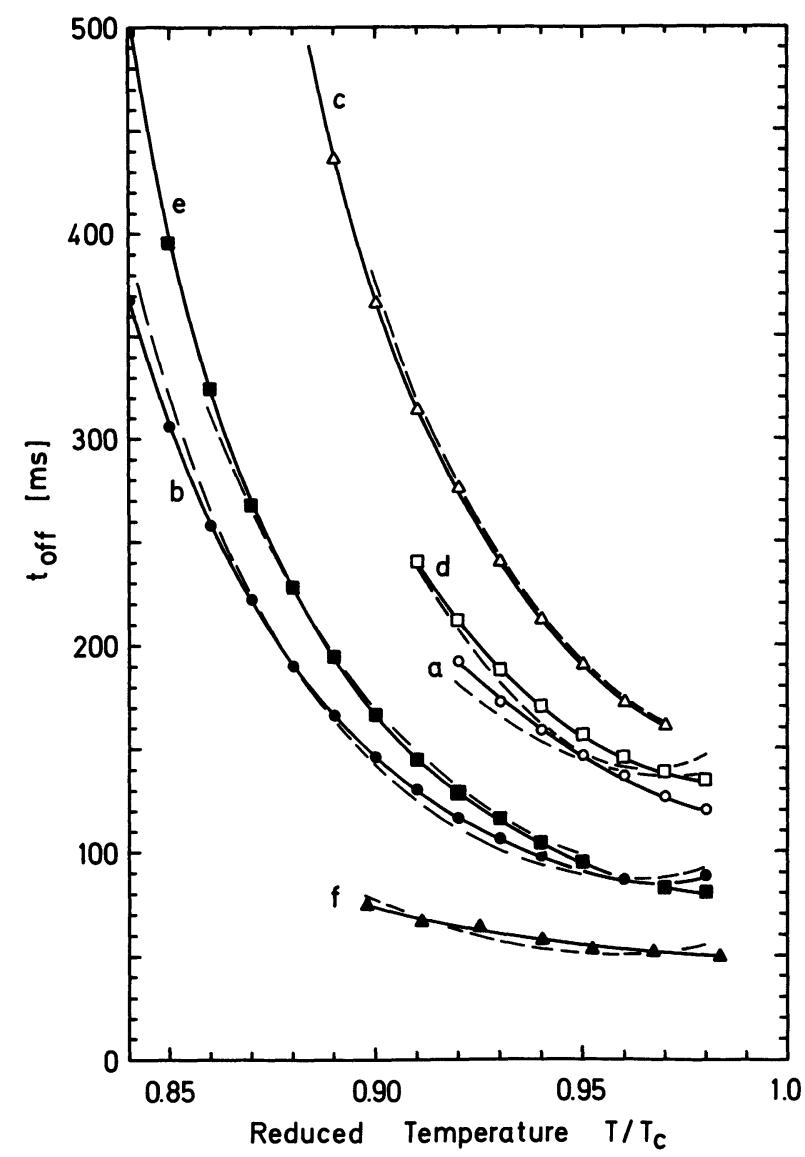

Fig. 9. - Measured (solid lines) and calculated (dotted lines) temperature dependence of the turn-off times of TN-LCDs using mixtures $a-f$. The calculated graphs were obtained from the measurements of $\eta$ and $k_{i i}$ using equation (12) and the scaling factors $f_{\text {off }}$ depicted in table III. 
Table III. - Measured $\left(t_{\mathrm{on}}, t_{\mathrm{off}}\right)$ and calculated $\left(t_{\mathrm{on}}^{*}, t_{\mathrm{off}}^{*}\right)$ turn-on and turn-off times of TN-LCDs. $f_{\text {on }}, f_{\text {off }}=s c a l$ ing factors (see text), $\theta=$ bias tilt angle measured at $22^{\circ} \mathrm{C}$. All data depicted in table $\mathrm{III}$ - except for $\theta$ - were determined at constant temperature $\left(T_{\mathrm{c}}-T\right)=20^{\circ} \mathrm{C}$.

\begin{tabular}{|c|c|c|c|c|c|c|c|c|}
\hline Mixture & $\begin{array}{c}t_{\text {on }} \\
(\mathrm{ms})\end{array}$ & $\begin{array}{c}t_{\mathrm{on}}^{*} \\
(\mathrm{~ms})\end{array}$ & $\begin{array}{c}t_{\text {off }} \\
(\mathrm{ms})\end{array}$ & $\begin{array}{c}t_{\text {off }}^{*} \\
(\mathrm{~ms})\end{array}$ & $f_{\text {on }}$ & $f_{\text {off }}$ & $\theta$ & $\begin{array}{c}\eta / \Delta \varepsilon \\
\text { (cp) }\end{array}$ \\
\hline- & - & - & - & - & - & - & - & - \\
\hline$a$ & 23.8 & 22.8 & 162 & 155 & 10.43 & 3.20 & $22.2^{\circ}$ & 1.42 \\
\hline$b$ & 27.5 & 29.0 & 96 & 92 & 14.39 & 3.53 & $23.2^{\circ}$ & 1.23 \\
\hline$c$ & 42.6 & 43.5 & 214 & 213 & 15.53 & 3.79 & $21.6^{\circ}$ & 1.74 \\
\hline$d$ & 59.0 & 52.0 & 176 & 169 & 11.35 & 3.27 & $24.0^{\circ}$ & 2.73 \\
\hline$e$ & 27.0 & 33.2 & 102 & 104 & 12.05 & 2.89 & $23.4^{\circ}$ & 1.64 \\
\hline$f$ & 33.9 & 33.9 & 59 & 52 & 14.43 & 3.32 & $21.6^{\circ}$ & 1.34 \\
\hline
\end{tabular}

The bias tilt angles $\theta$ measured for each mixture at $22{ }^{\circ} \mathrm{C}$ are depicted in table III. $t_{\text {on }}$ and $t_{\text {off }}$ were calculated from the measurements of $\Delta \varepsilon$ (Fig. 4), $k_{i i}$ (Fig. 6) and $\eta$ (Fig. 3) using equations (11) and (12) respectively. The calculated values of $t_{\text {on }}$ and $t_{\text {off }}$ were fitted to the response-time measurements (Figs. 8 and 9) by using one scaling factor each for every mixture. The scaling factors used are depicted in table III. Table III also shows the measured $\left(t_{\text {on }}\right.$, $\left.t_{\text {off }}\right)$ and the calculated $\left(t_{\text {on }}^{*}, t_{\text {off }}^{*}\right)$ response times at $\left(T_{\mathrm{c}}-T\right)=20^{\circ} \mathrm{C}$ for mixtures $a-f$ using the material constants depicted in Tables I and II respectively for the calculations.

The factors $f_{\text {on }}$ (Table III) show that the actually calculated turn-on times obtained from the measurements of $\Delta \varepsilon$ and $k_{i i}$ using equation (11) are 10-15 times shorter than those obtained from responsetime measurements in TN-LCDs. A similar discrepancy - which is probably due to the small angle approximation used in deriving equation (11) was recently reported for measurements made with more complex mixtures at room temperature [6]. However, figure 8 shows an astonishingly good agreement between the temperature dependence of measured and calculated rise-times for all mixtures except for temperatures $\left(T_{\mathrm{c}}-T\right) \lesssim 5^{\circ} \mathrm{C}$. The deviations occurring upon approaching the nematic-isotropic transition temperature may at least partly be due to the onset of temperature dependence of bias tilt in this temperature region (Fig. 2). The surprisingly small variations $(<30 \%)$ of the scaling factors $f_{\text {on }}$, whereas $\bar{f}_{\text {on }}=13.03$ being the averaged scaling factor, used to fit the measured with the calculated temperature dependence of $t_{\text {on }}$ (Table III, Fig. 8) shows, that the relative temperature dependence of the rise-times of markedly different LCclasses can be correlated with their dielectric and elastic properties using equation (11).

The finding that $t_{\text {on }}$ of the viscous ester mixture $c$ is noticeably lower than that of biphenyl mixture $d$ (Table III) confirms our previous results [6] which showed that the turn-on times of dielectrically strongly anisotropic substances tend to be short. This is due to the decrease of the ratio $\eta / \Delta \varepsilon \propto t_{\text {on }}$ with increasing $\Delta \varepsilon$ (Table III). Thus $\eta / \Delta \varepsilon$ gives a good indication of the relative turn-on behaviour of different LC-materials in TN-LCDs.

The graphs depicted in figure 9 show the good agreement of the temperature dependence between measured and calculated turn-off times over the whole mesomorphic range of mixtures $a-d$. Smaller scaling factors $f_{\text {off }}\left(f_{\text {off }}=2.9-3.8\right)$ had to be used compared with those fitting the calculated turn-on times (Table III). The variations of $f_{\text {off }}$ between the different mixtures were also smaller $(<25 \%$, Table III). From table III one gets an average scaling factor $\bar{f}_{\text {off }}=3.4$. An even better agreement might be achieved if boundary effects $[28,29]$ (bias tilt, boundary coupling) were taken into account.

The good agreement found between the temperature dependence of measured and calculated turnoff times (Fig. 9) implies that the temperature dependence of $t_{\text {off }}$ in TN-LCDs is mainly determined by the temperature dependence of the viscosity. This follows from the measured strong temperature dependence of $\eta$ (Fig. 3) compared with that of $k(T)$. Thus LCmaterials with low activation energies $A$ (4) - such as pyrimidines or PCHEs (Table I) - exhibit also a small dependence of $t_{\text {off }}$ versus temperature when used in TN-LCDs. Whereas temperature compensation for $t_{\text {on }}$ in TN-LCDs could best be achieved - due to $t_{\text {on }} \propto \eta / \Delta \in-$ by LC-materials exhibiting similar temperature dependence for $\eta(T)$ and $\Delta \varepsilon(T)$.

5. Conclusions. - We have measured the temperature dependence of the viscous, the dielectric, the optical and the elastic properties of six binary mixtures belonging to six different positive dielectric liquid crystal classes. The binary mixtures each contained a pentyl and a heptyl component in molar proportions $40 \%: 60 \%$. The components used were Schiff bases, alkyl and alkoxy biphenyls, esters, phenylpyrimidines and hydrogenated esters. The complete sets of material constants - determined for most of the LC-classes investigated for the first time - were correlated with the electro-optical performance of these substances in twisted-nematic displays. Surpri- 
singly good agreement was found between classspecific material properties and the temperature dependence of the response times in TN-LCDs. It was further shown that the multiplexing behaviour of the liquid crystals investigated is qualitatively in agreement with their splay and bend elastic properties.

The experiments show that the temperature dependence of rise and decay times $t_{\text {on }}$ and $t_{\text {off }}$ of TN-LCDs can be expressed over most of the mesomorphic range, by $t_{\text {on }} \propto \eta / \Delta \varepsilon$ and $t_{\text {off }} \propto \eta / k$ respectively, regardless of the chemically considerably different materials used. This also confirms our previous findings which have indicated that the experimentally easily accessible bulk viscosity which was found to depend linearly on the viscosity coefficients $\eta_{1}, \eta_{2}$ and $\eta_{3}$ gives a good indication of the relative viscous behaviour of different LC-materials. Yet, deviations were found when approaching the nematic-isotropic transition temperature. This may have been due to the onset of temperature dependence of bias tilt angle observed with various liquid crystal classes when using large bias tilt displays. However, no temperature depen- dence of bias tilt was found in low bias tilt $\left(\theta<0.5^{\circ}\right)$ displays. This is a prerequisite when measuring elastic properties of LC-materials in homogeneously aligned samples.

The maximum number of multiplexable lines $N_{\max }$ of a TN-LCD matrix was found to depend qualitatively on the splay/bend ratio $k_{33} / k_{11} . N_{\text {max }}$ increased with decreasing ratio $k_{33} / k_{11}$. The best multiplexing performance among the pentyl/heptyl mixtures investigated was found for pyrimidines. However, still better multiplexing performance can probably be achieved when using shorter chain molecules for which the ratio $k_{33} / k_{11}$ is likely to decrease [4]. This is being further investigated using various homologous series of LC-materials.

Acknowledgments. - We are very much indebted to A. Boller and A. Villiger who synthesized the LCcomponents used. We also gratefully acknowledge the assistance of B. Blöchliger and R. Ayer in the experimental work.

\section{References}

[1] Karat, P. P. and Madhusudana, N. V., Mol. Cryst. Liq. Cryst. 36 (1976) 51.

[2] Schadt, M. and Müller, F., J. Chem. Phys. 65 (1976) 2224.

[3] De Jeu, W. H., Claassen, W. A. P. and Spruijt, A. M. J., Mol. Cryst. Liq. Cryst. 37 (1976) 269.

[4] De Jeu, W. H. and Claassen, W. A. P., J. Chem. Phys. 67 (1977) 3705.

[5] Gruler, H., SChefFer, T. J. and Meier, G., Z. Naturforsch. 27a (1972) 966.

[6] Schadt, M. and Müller, F., IEEE Trans. Electron Devices ED-25 (1978) 1125

[7] Schadt, M. and Helfrich, W., Appl. Phys. Lett. 18 (1971) 127.

[8] Schadt, M., J. Chem. Phys. 56 (1972) 1494.

[9] Maze, C. and Johnson, D., Mol. Cryst. Liq. Cryst. 33 (1976) 213.

[10] Guyon, E., Pieranski, P. and Boix, M., Lett. Appl. Eng. Sci. 1 (1973) 19.

[11] Toriyama, K. and Ishibashi, T., in Nonemissive Electrooptic Displays, ed. by Kmetz A. R. and v. Willisen F. K. (Plenum, New York) 1976.

[12] Scheffer, T. J. and Nehring, J., J. Appl. Phys. 48 (1977) 1783.

[13] Schadt, M. and v. Planta, C., J. Chem. Phys. 63 (1975) 63.

[14] Toda, A., Mada, H. and Kobayashi, S., Japan J. Appl. Phys. 17 (1978) 261.
[15] Kahn, F. J., Mol. Cryst. Liq. Cryst. 38 (1977) 109.

[16] Boller, A., Cereghetti, M., Schadt, M. and Scherrer, H., Mol. Cryst. Liq. Cryst. 42 (1977) 215.

[17] Gray, G. W., Harrison, K. J. and Nash, J. A., Electron Lett. 9 (1973) 130.

[18] Kuschel, F., König, S., Kresse, H., Pfeiffer, D., WiegeleBEN, A., Wulf, J. and Demus, D., Z. Chem. 17 (1977) 62.

[19] Emenschink, R., Erdmann, D., Krause, J. and Pohl, L., Angew. Chem. 89 (1977) 103.

[20] MaIER, W. and MeIer, G., Z. Naturforsch. 16a (1961) 262.

[21] MaIER, W. and SAUPE, A., Z. Naturforsch. 14a (1959) 882.

[22] Alt, P. M. and PleshKo, P., IEEE Trans. Electron Devices 21 (1974) 146.

[23] KMETz, A. R., Proceedings of the SID Meeting of the IEEE held in San Francisco, April (1978).

[24] Berreman, D. W., J. Opt. Soc. Am. 63 (1973) 1374.

[25] Baur, G., Windscheid, F. and Berreman, D. W., Appl. Phys. A 8 (1975) 101

[26] TARry, H. A., SERL. Techn. J. 25 (1975) 1.

[27] Jakeman, E. and Raynes, E. P., Phys. Lett. A 39 (1974) 12.

[28] MeYerhofer, D., J. Appl. Phys. 48 (1977) 1179.

[29] Nehring, J., Kmetz, A. R. and SCheffer, T. J., J. Appl. Phys. 47 (1976) 850. 